\title{
Análise da associação entre adesão terapêutica e letramento em saúde em hipertensos
}

\author{
Analysis of the association between therapeutic membership and health literacy in hypertensive \\ patients
}

\section{Análisis de la asociación entre adherencia terapéutica y alfabetización de salud en hipertensos}

\section{RESUMO}

Objetivo: Analisar a associação entre adesão ao tratamento e letramento funcional em saúde em hipertensos. Métodos: Estudo transversal, descritivo, realizado na atenção primária em saúde, com 242 hipertensos. Na coleta de dados, foram utilizados o Questionário de adesão ao tratamento da hipertensão arterial e o Morisky Medication Adherence Scale ambos sobre adesão terapêutica e o Short Assessment of Health Literacy for Portuguese-Speaking Adults sobre letramento em saúde. Para a análise descritiva e inferencial, foi usado o software IBM SPSS ${ }^{\circledR}$, com significância estatística de 5\%. Resultados: Os níveis de adesão dos questionários foram diferentes e o letramento em saúde inadequado, sem associação significativa. Conclusão: O letramento funcional em saúde demonstrou alguma influência na adesão, mesmo sem associação significativa, devendo ser considerada na promoção da adesão e na redução de riscos a hipertensos na atenção primária à saúde.

Descritores: Hipertensão; Letramento em Saúde; Cooperação e Adesão ao Tratamento; Comportamentos Relacionados com a Saúde.

\section{ABSTRACT}

Objective: To analyze the association between treatment adherence and functional health literacy in hypertensive patients. Methods: A cross-sectional, descriptive study, carried out in primary health care, with 242 hypertensive patients. In the data collection, the Questionnaire on adherence to the treatment of arterial hypertension and the Morisky Medication Adherence Scale were used, both on therapeutic adherence and the Short Assessment of Health Literacy for Portuguese-Speaking Adults on health literacy. For descriptive and inferential analysis, the IBM SPSS ${ }^{\circledR}$ software was used, with a statistical significance of 5\%. Results: The levels of adherence to the questionnaires were different and health literacy was inadequate, with no significant association. Conclusion: Functional health literacy has shown some influence on adherence, even without a significant association, and should be considered in promoting adherence and reducing risks to hypertensive patients in primary health care.

Descriptors: Hypertension; Health Literacy; Treatment Adherence and Compliance; Health Behavior.

\section{RESUMEN}

Objetivo: Analizar la asociación entre la adherencia al tratamiento y la alfabetización funcional de salud en hipertensos. Métodos: Estudio descriptivo transversal, realizado en atención primaria de salud, con 242 hipertensos. En la recogida de datos se utilizó el Cuestionario de adherencia al tratamiento de la hipertensión arterial y la Escala de adherencia a la medicación de Morisky, sobre adherencia terapéutica y la evaluación corta de alfabetización en salud. Para el análisis descriptivo e inferencial se utilizó el software IBM SPSS ${ }^{\circledR}$, con significancia estadística 5\%. Resultados: los niveles de adherencia a los cuestionarios fueron diferentes y la alfabetización en salud fue inadecuada, sin asociación significativa. Conclusión: La alfabetización en salud funcional ha mostrado cierta influencia en la adherencia, incluso sin una asociación significativa, y debe considerarse para promover la adherencia y reducir los riesgos para los pacientes hipertensos en la atención primaria de salud.

Descriptores: Hipertensión; Alfabetización en Salud; Cumplimiento y Adherencia al Tratamiento; Conductas Relacionadas con la Salud.

\begin{tabular}{|c|}
\hline $\begin{array}{r}\text { Aline Castelo Girão }{ }^{1} \\
\text { 0000-0002-1028-1286 } \\
\end{array}$ \\
\hline $\begin{array}{r}\text { Thereza Maria Magalhães } \\
\text { Moreira }^{1} \\
0000-0003-1424-0649 \\
\end{array}$ \\
\hline $\begin{array}{l}\text { Juliana Rodrigues da Silva }{ }^{1} \\
0 \text { 0000-0001-7267-8775 }\end{array}$ \\
\hline $\begin{array}{l}\text { Emiliana Bezerra Gomes }{ }^{2} \\
\text { 0000-0002-7135-512X }\end{array}$ \\
\hline $\begin{array}{l}\text { Gilliane Ferreira da Silva }{ }^{1} \\
0000-0003-2700-8339 \\
\end{array}$ \\
\hline $\begin{array}{l}\text { Maria Lúcia Duarte Pereira }{ }^{1} \\
0000-0003-0079-5248\end{array}$ \\
\hline $\begin{array}{r}\text { Jênifa Cavalcante dos Santos } \\
\text { Santiago } \\
\text { (1) 0000-0001-9815-8698 }\end{array}$ \\
\hline $\begin{array}{l}1 \text { Universidade Estadual do Ceará, CE, } \\
\text { Brasil. } \\
2 \text { Universidade Regional do Cariri, CE, } \\
\text { Brasil. }\end{array}$ \\
\hline
\end{tabular}

Autor correspondente: Aline Castelo Girão E-mail: alinecastelog@hotmail.com 


\section{INTRODUÇÃO}

A hipertensão arterial sistêmica (HAS) tem elevada prevalência nos países em desenvolvimento. A assintomatologia de sua fase inicial falta de informação populacional e dificuldades no acesso aos serviços de saúde colaboram para seu baixo controle ${ }^{(1)}$.

A baixa adesão ao tratamento causa prejuízos no atendimento às pessoas com HAS, pois aumenta custos sociais, absenteísmo no trabalho, licenças para tratamento de saúde e aposentadorias por invalidez $z^{(2)}$.

A média de adesão ao tratamento de doenças crônicas, como a HAS, em países desenvolvidos, é de $50 \%$, sendo menor nos países em desenvolvimento ${ }^{(3)}$. Ademais, a adesão terapêutica ao tratamento da HAS é multifatorial e um desafio aos profissionais da saúde, reiterando a relevância do desenvolvimento de estudos sobre o cotidiano desses pacientes e sobre estratégias exitosas a eles aplicáveis ${ }^{(4)}$.

A abordagem terapêutica inclui medidas não farmacológicas e anti-hipertensivos para o controle da pressão arterial (PA), proteção de órgãos-alvo e de desfechos cardiovasculares e renais. Muitas vezes, ações não farmacológicas são descontinuadas pela incompreensão de informações, durante as consultas ${ }^{(5)}$ e déficit no Letramento Funcional em Saúde (LFS), gerando dificuldade no cuidado, sobretudo, em cronicidades $^{(6)}$.

A capacidade do hipertenso em aderir ao tratamento perpassa pelo seu nível de letramento em saúde, pois a capacidade de compreensão das informações sobre seu tratamento é altamente significativa para a manutenção da saúde e prevenção de agravos. Estudos mostram que o letramento contribui para a adesão ao uso de medicações, o controle da pressão arterial, a prática de atividade física e a redução da mortalidade ${ }^{(7-8)}$.

Nesse contexto, o enfermeiro se destaca na educação em saúde, e entender o nível de letramento em saúde de pessoas em adoecimento crônico é essencial para subsidiar a sistematização da assistência de enfermagem. A inserção do diagnóstico de enfermagem "Disposição para letramento em saúde melhorado", na NANDA International no ano de 2016, possibilitou incluir pelo julgamento clínico da motivação do indivíduo em aumentar o letramento em saúde, maior especificidade sobre essa questão no Processo de enfermagem e delinear intervenções que melhorem o letramento e, consequentemente, reduzam os riscos à saúde e melhorem a qualidade de vida pela promoção do autocuidado e maior adesão ao tratamento da $\operatorname{HAS}^{(9-10)}$

Portanto o LFS é um conceito relevante, principalmente nos aspectos que envolvem o processo saúde-doença da população e o cuidado de enfermagem, no campo de promoção da saúde, possibilitando às pessoas com hipertensão um posicionamento ativo quanto às questões da própria saúde, incluindo, a capacidade de mudança na sua própria condição de saúde, da família e da comunidade ${ }^{(9)}$.

Assim, diante do exposto, o conhecimento da associação entre a adesão ao tratamento antihipertensivo e o letramento funcional em saúde poderia direcionar a assistência de enfermagem à promoção da saúde em serviços de atenção primária à saúde. Portanto foi objetivo do estudo analisar a associação entre adesão ao tratamento e letramento funcional em saúde em hipertensos.

\section{MÉTODOS}

Estudo transversal, em uma unidade de Atenção primária à Saúde (APS), em FortalezaCeará, com hipertensos cadastrados e acompanhados rotineiramente para consultas. O serviço oferece atendimento informatizado e prontuário eletrônico, farmácia abastecida com fármacos da atenção primária, coleta diária de exames laboratoriais, atendimento odontológico com sala de raio-x e ambientes climatizados. O estudo foi aprovado pelo Comitê de Ética em Pesquisa (CEP) da Universidade Estadual do Ceará, sob o parecer no 3.438 .816 e seguiu as orientações da Resolução 466/2012 do Conselho Nacional de Saúde, que diz respeito à pesquisa com seres humanos ${ }^{(11)}$. Após esclarecimento sobre o conteúdo da pesquisa e sigilo das identidades, foi solicitado aos participantes a assinatura do Termo de Consentimento Livre e Esclarecido (TCLE).

A coleta dos dados ocorreu, por meio de fonte primária, diretamente com os pacientes, aplicando-se questionários validados no país, nos dias de segunda a sexta feira, nos turnos manhã e tarde, de acordo com a disponibilidade de cada pesquisadora, sendo realizada por três pesquisadoras capacitadas. Entre os dados clínicos, a medida da pressão arterial foi realizada pela técnica auscultatória com o esfigmomanômetro aneroide adulto da marca 
SOLIDOR $^{\circledast}$ pelo profissional auxiliar de enfermagem da unidade.

A população foi de 1500 hipertensos cadastrados em atendimento na unidade. No cálculo da amostra para a população finita ${ }^{(12)}$, adotou-se prevalência de adesão de $25 \%$ e erro de 5\%, resultando em 242 hipertensos. Foi considerada a significância de $5 \%(p<0,05)$.

Foram adotados como critérios de inclusão na amostra: diagnosticado com HAS, cadastrado na unidade, com $\geq 18$ anos de idade, lê e escreve, em tratamento anti-hipertensivo prescrito (farmacológico ou não) e reside na zona urbana do município. Foram critérios de exclusão: a) surdo/mudo; b) gestantes; c) diabéticos; d) mudar de município durante coleta. A seleção dos participantes se deu por conveniência, sendo abordados os hipertensos no dia de suas consultas.

Foram aplicados três questionários: Questionário de adesão ao tratamento da HAS (QATHAS) e Morisky Medication Adherence Scale (MMAS-8) sobre adesão terapêutica e o Short Assessment of Health Literacy for PortugueseSpeaking Adults (SAHLPA-18) sobre LFS.

O Questionário de Adesão ao Tratamento da HAS (QATHAS) ${ }^{(13)}$ classifica a adesão terapêutica geral, considerando o tratamento farmacológico e não farmacológico, é um instrumento elaborado e validado com hipertensos em Fortaleza, no Estado do Ceará, e se mostrou adequado para avaliar a adesão ao tratamento da HAS. Ele situa o respondente em uma escala de adesão que varia de 60 a 110, pela qual o profissional de saúde saberá, com exatidão, em quais aspectos do tratamento o hipertenso deverá ser mais cuidadoso para ascender na escala. Diferente da Escala de Adesão Terapêutica de Morisky (MMAS-8) ${ }^{(14)}$ que considera, na sua medida, apenas o tratamento farmacológico, justifica o uso de dois questionários para a mensuração da adesão no estudo. O MMAS-8 usada é uma versão em português, traduzida e validada no Brasil e que avalia a adesão medicamentosa, em que o paciente deve responder a um questionário de breves perguntas posteriormente pontuadas e classificadas. As especificidades das escalas, quanto à adesão nos aspectos medicamentosos e não medicamentosos e sua relação com o letramento, pareceram uma oportunidade de eleger uma escala, pela existência ou não de divergências entre elas e o letramento em saúde, dada a inexistência de um padrão ouro.

Para a avaliação do LFS, o instrumento aplicado foi adaptado e validado por pesquisadores brasileiros para estimar o nível de alfabetismo em saúde de adultos.

Considerou-se aderente pelo QATHAS pessoas $\geq 90$ pontos $^{(13)}$. Já pelo MMAS-8 (versão 2 da escala de avaliação da adesão medicamentosa, validada no Brasil), a adesão se divide em alta ( 8 pontos), média (6-7 pontos) e baixa(<6 pontos), sendo adequado somente se alcançar a pontuação máxima de oito pontos ${ }^{(14)}$.

Já o LFS foi aferido pelo Short Assessment of Health Literacy for Portuguese-Speaking Adults (SAHLPA-18), que varia de 0 a 18, sendo correto o item se há acerto de pronúncia e associação palavras-significado. Resultado 0-14 sugere LFS inadequado e 15-18 adequado ${ }^{(15)}$.

A análise dos dados se deu no software Statistical Package for the Social Sciences (IBM SPSS $^{\circledR}$ ) versão 20.0, com análise de frequências absoluta e relativa das variáveis categóricas, uso de medidas de tendência central (média/mediana) e dispersão (desvio-padrão, mínimo e máximo) para variáveis numéricas. A análise estatística inferencial se deu com o teste Qui-quadrado de Pearson $(X)^{2}$, razão de prevalência e razão de chances.

\section{RESULTADOS}

A idade variou de 18-95 anos, com amplitude de 77 anos. A média das idades foi de $59,78$ ( $\pm D P: 11,666)$, moda 56 anos e mediana 60,5 anos. Do total, 48,4\% eram adultos (18-59 anos) e 51,6\% idosos (60-95 anos). Houve predomínio de mulheres $(68,2 \%)$. A maioria apontou baixa escolaridade $(68,6 \%)$ com ensino fundamental completo ou não e renda mensal $\leq 1$ salário mínimo (SM) $(39,6 \%)$, comumente para sustentar até cinco pessoas por domicílio $(93,8 \%)$. Sobre características clínicas, $76,5 \%$ estavam obesos e 55\% tinham PA normal/pré-hipertensão e $45 \%$ com PA elevada, estando presente em $31,4 \%$ das mulheres ( $n=180$ ) e $68,6 \%$ dos homens $(n=62)$.

A adesão ao tratamento pelo QATHAS e MMAS-8 e o LFS foram avaliados, e foi verificada sua associação com variáveis sociodemográficas e clínicas. Pelo QATHAS, a maioria $(87,2 \%)$ aderiu ao tratamento ( $\geq 90)$, mas apenas $5,4 \%$ alcançaram nível máximo de adesão $(110=$ tomar antihipertensivo adequadamente, comer sem sal e 
seguir o tratamento não medicamentoso). Pelo QATHAS ser novo, porém mais amplo, a adesão foi verificada também com o MMAS-8, que mede adesão farmacológica.

Pelo instrumento MMAS-8, 38,4\% tinham baixa adesão e $61,6 \%$ média/alta adesão. Portanto resultados diferentes, mas não divergentes, pois o QATHAS mede adesão farmacológica e não farmacológica, sendo mais completa a adesão medida pelo QATHAS.
A Tabela 1 resume a associação entre a adesão terapêutica dos hipertensos com as variáveis analisadas.

Entrecruzaram-se variáveis independentes (faixa etária, sexo, escolaridade, renda, PA, IMC, LFS) com variável dependente (adesão). Pelo QATHAS, apenas PA teve associação estatística com adesão $(p=0,020)$. Pelo MMAS-8 houve associação significativa entre adesão e idade $(p=0,024)$.

Tabela 1 - Associação entre adesão e variáveis sociodemográficas e clínicas (n=242). Fortaleza-Ceará-Brasil, 2019

\begin{tabular}{|c|c|c|c|c|c|c|c|}
\hline \multirow{2}{*}{ Variáveis } & \multirow{2}{*}{$\begin{array}{l}\text { Total } \\
\mathrm{fi}^{+}(\%)\end{array}$} & \multicolumn{2}{|c|}{ ADESÃO QATHAS ${ }^{\S}$} & \multirow{2}{*}{$\begin{array}{c}\text { p-valor } \\
\text { OR" (IC 95\%)** }\end{array}$} & \multicolumn{2}{|c|}{ ADESÃO MMAS-8|। } & \multirow{2}{*}{$\begin{array}{c}\text { p-valor } \\
\text { OR (IC 95\%) }\end{array}$} \\
\hline & & $\begin{array}{l}\text { Adere } \\
\text { fi (\%) }\end{array}$ & $\begin{array}{l}\text { Não adere } \\
\text { fi (\%) }\end{array}$ & & $\begin{array}{l}\text { Alta/Média } \\
\text { fi (\%) }\end{array}$ & $\begin{array}{l}\text { Baixa } \\
\text { fi (\%) }\end{array}$ & \\
\hline \multicolumn{8}{|l|}{ Faixa etária } \\
\hline$<60$ anos & $117(48,3 \%)$ & $99(40,9 \%)$ & $18(7,4 \%)$ & 0,246 & $64(26,4 \%)$ & $53(21,9 \%)$ & $0,024^{*}$ \\
\hline$\geq 60$ anos & $125(51,7 \%)$ & $112(46,3 \%)$ & $13(5,4 \%)$ & $0,638(0,298-1,369)$ & $86(35,5 \%)$ & $39(16,1 \%)$ & $0,548(0,324-0,926)$ \\
\hline Sexo & & & & 0,641 & & & \\
\hline Masculino & $62(25,6 \%)$ & $53(21,9 \%)$ & $9(3,7 \%)$ & $1,220(0,529-2,813)$ & $37(15,3 \%)$ & $25(10,3 \%)$ & 0,664 \\
\hline Feminino & $180(74,4 \%)$ & $158(65, \%)$ & $22(9,1 \%)$ & & $113(46,7 \%)$ & $67(27,7 \%)$ & $1,140(0,631-2,057)$ \\
\hline \multicolumn{8}{|l|}{ Escolaridade } \\
\hline Ensino Fundamental & $166(68,6 \%)$ & $146(60,3 \%)$ & $20(8,3 \%)$ & 0,600 & $100(41,3 \%)$ & $66(27,3 \%)$ & 0,409 \\
\hline Ensino Médio/ & & & & $1,235(0,560-2,727)$ & & & $0,788(0,447-1,389)$ \\
\hline superior & $76(31,4 \%)$ & $65(26,9 \%)$ & $11(4,5 \%)$ & & $50(20,7 \%)$ & $26(10,7 \%)$ & \\
\hline \multicolumn{8}{|l|}{ Renda Familiar } \\
\hline Até 1 Salário mínimo & $85(35,1 \%)$ & $74(30,6 \%)$ & $11(4,6 \%)$ & 0,964 & $46(19 \%)$ & $39(16,1 \%)$ & 0,064 \\
\hline 1 |- 5 Salário mínimo & $157(64,9 \%)$ & $137(56,6)$ & $20(8,3 \%)$ & $0,982(0,442-2,160)$ & $104(43 \%)$ & $53(21,9 \%)$ & $0,601(0,350-1,031)$ \\
\hline $\begin{array}{l}\text { Pessoas/ } \\
\text { domicilio }\end{array}$ & & & & 0,459 & & & 0,484 \\
\hline Até 5 pessoas & $226(93,8 \%)$ & $196(81,3 \%)$ & $30(12,4 \%)$ & $0,467(0,59-3,679)$ & $141(58,5 \%)$ & $85(35,3 \%)$ & $0,451(0,508-4,146)$ \\
\hline$>5$ pessoas & $15(6,2 \%)$ & $14(5,8 \%)$ & $1(0,4 \%)$ & & $8(3,3 \%)$ & $7(2,9 \%)$ & \\
\hline \multicolumn{8}{|l|}{$\mathrm{IMC}^{\ddagger}$} \\
\hline Peso & $57(23,6 \%)$ & $51(21,1 \%)$ & $6(2,5 \%)$ & 0,555 & $37(15,3 \%)$ & $20(8,3 \%)$ & 0,602 \\
\hline baixo/adequado & & & & $1,328(0,516-3,417)$ & & & $1,179(0,635-2,189)$ \\
\hline $\begin{array}{l}\text { Sobrepeso/ } \\
\text { Obesidade }\end{array}$ & $185(76,4 \%)$ & $160(66,1 \%)$ & $25(10,3 \%)$ & & $113(46,7 \%)$ & $72(29,8 \%)$ & \\
\hline \multicolumn{8}{|l|}{ PA } \\
\hline $\begin{array}{l}\text { Normal/pré- } \\
\text { hipertenso }\end{array}$ & $133(55 \%)$ & $122(50,4 \%)$ & $11(4,5 \%)$ & $\begin{array}{c}0,020^{*} \\
2,492(1,137-5,463)\end{array}$ & $86(35,5 \%)$ & $47(19,4 \%)$ & $\begin{array}{c}0,343 \\
1287(0764-2167)\end{array}$ \\
\hline Hipertensão & $109(45 \%)$ & $89(36,8 \%)$ & $20(8,3 \%)$ & & $64(26,4 \%)$ & $45(18,6 \%)$ & \\
\hline
\end{tabular}

Fonte: Dados da pesquisa.

Nota: $\mathrm{p}^{*}$ = significância estatística; $\mathrm{fi}^{\dagger}=$ Frequência absoluta; $I M C^{\ddagger}=$ Índice de massa corporal; QATHAS $=$ Questionário de adesão ao tratamento da Hipertensão Arterial Sistêmica; MMAS-8II= Morisky Medication Adherence Scale; ORף= Odds Ratio; IC**=Intervalo de Confiança.

Sobre LFS, cada item correto acertava pronúncia e associação, valia um ponto, sendo no máximo 18. LFS inadequado era 0-14 e adequado de15-18 $8^{(15)}$. A maioria obteve 0-14 pontos, sendo a mediana de acertos $13,39( \pm D P 3,26)$, ou LFS inadequado. Do total, $42,2 \%$ dos hipertensos tiveram LFS adequado e 57,8\% inadequado.

A variável adesão terapêutica analisada pelos instrumentos QATHAS e MMAS8/escala Morisky não teve associação estatística significativa com o LFS, quando adesão mensurada via QATHAS $(p=0,232)$ ou MMAS8/escala Morisky ( $p=0,835)$ (Tabela 2).

Apesar de não evidenciada a associação entre o letramento e a adesão, as pessoas hipertensas e a comunidade em geral devem ser informadas e educadas, quanto aos fatores relacionados à adesão e que podem influenciar no letramento, optando ou não conscientemente por uma vida mais saudável. 
Tabela 2 - Associação entre letramento funcional em saúde e variáveis sociodemográfica, clínicas e adesão (n=242). Fortaleza-CearáBrasil, 2019

\begin{tabular}{|c|c|c|c|c|c|c|}
\hline \multirow{2}{*}{\multicolumn{2}{|c|}{ Variáveis }} & \multirow{2}{*}{\multicolumn{2}{|c|}{ Total }} & \multicolumn{2}{|c|}{ LFS } & \multirow{3}{*}{ p-valor } \\
\hline & & & & \multirow{2}{*}{$\begin{array}{c}\text { Adequado } \\
\text { fi (\%) }\end{array}$} & \multirow{2}{*}{$\begin{array}{c}\text { Inadequado } \\
\text { fi (\%) }\end{array}$} & \\
\hline & & $\mathrm{fi}^{+}$ & $\%$ & & & \\
\hline \multirow{2}{*}{ Sexo } & Feminino & 180 & 68,2 & $78(32,23 \%)$ & $102(42,15 \%)$ & $>0,05$ \\
\hline & Masculino & 62 & 23,5 & $24(9,92 \%)$ & $38(15,70 \%)$ & \\
\hline \multirow{2}{*}{ Faixa etária } & $<60$ anos & 117 & 48,3 & $57(23,55 \%)$ & $60(24,79 \%)$ & $<0,05^{*}$ \\
\hline & 60-95 anos & 125 & 51,7 & $45(18,60 \%)$ & $80(33,06 \%)$ & \\
\hline \multirow{2}{*}{ Escolaridade } & Ensino Fundamental & 166 & 68,6 & $51(21,07 \%)$ & $115(47,52 \%)$ & $<0,05^{*}$ \\
\hline & Ensino Médio/Superior & 76 & 31,4 & $51(21,07 \%)$ & $25(10,33 \%)$ & \\
\hline \multirow{2}{*}{ Renda } & <1 Salário mínimo & 96 & 39,7 & $8(3,31 \%)$ & $17(7,02 \%)$ & $>0,05$ \\
\hline & 1 - 5 Salários mínimos & 146 & 60,3 & $94(38,84 \%)$ & $123(50,83 \%)$ & \\
\hline \multirow{2}{*}{ Pessoas no domicílio } & $1-2$ pessoas & 88 & 36,4 & $37(15,29 \%)$ & $51(21,07 \%)$ & $>0,05$ \\
\hline & 3-5 pessoas & 154 & 63,6 & $65(26,86 \%)$ & $89(36,78 \%)$ & \\
\hline \multirow{2}{*}{$\mathrm{IMC}^{\ddagger}$} & Peso normal/baixo & 57 & 23,5 & $20(8,26 \%)$ & $37(15,29 \%)$ & $>0,05$ \\
\hline & Sobrepeso/Obesidade & 185 & 76,5 & $82(33,88 \%)$ & $103(42,56 \%)$ & \\
\hline \multirow{2}{*}{ Classe de PA } & Normal/ Pré-hipertensão & 133 & 55,0 & $62(25,62 \%)$ & $71(29,34 \%)$ & $>0,05$ \\
\hline & Hipertensão & 109 & 45,0 & $40(16,53 \%)$ & $69(28,51 \%)$ & \\
\hline \multirow{2}{*}{ Adesão-Moriskyll } & Alta/Média adesão & 149 & 61,6 & $64(26,45 \%)$ & $85(35,12 \%)$ & $>0,05$ \\
\hline & Baixa adesão & 93 & 38,4 & $38(15,70 \%)$ & $55(22,73 \%)$ & \\
\hline \multirow{2}{*}{ Adesão-QATHAS§ } & Adere & 211 & 87,2 & $92(38,02 \%)$ & $119(49,17 \%)$ & $>0,05$ \\
\hline & Não adere & 31 & 12,8 & $10(4,13 \%)$ & $21(8,68 \%)$ & \\
\hline
\end{tabular}

Fonte: Dados da pesquisa.

Nota: $\mathrm{p}^{*}=$ significância estatística; $\mathrm{fi}^{\dagger}=$ Frequência absoluta; IMC ${ }^{\ddagger}=$ Índice de massa corporal; QATHAS ${ }^{\S}=$ Questionário de adesão ao tratamento da Hipertensão Arterial Sistêmica; MMAS-8II= Morisky Medication Adherence Scale.

Faixa etária e escolaridade apresentaram significância estatística com LFS $(p<0,05)$. Os resultados mostraram LFS mais inadequado entre os mais velhos e com menor escolaridade.

\section{DISCUSSÃO}

No Brasil, hipertensos que mantêm PA<140x90mmHg (57,6\%) utilizam fármacos corretamente $(36,5 \%)$, aderem à terapêutica, alteram hábitos alimentares, abandonam vícios (tabagismo/alcoolismo) e incorporam atividades físicas ${ }^{(16-17)}$. Mas mudanças no estilo de vida e hábitos não são fáceis, o que pode explicar a diferença nos níveis de adesão obtidos com o QATHAS e MMAS-8, especialmente por esse último avaliar somente tratamento farmacológico.

Adesão ao tratamento da HAS deve incluir educação sobre o tema, capacidade de compreensão e interpretação das orientações de saúde para mudança no estilo de vida e adesão ao uso de fármacos, relacionados ao $\mathrm{LFS}^{(18)}$. A adesão ao tratamento é pauta de pesquisas, em todo o mundo, dado o desafio do seu manejo enquanto na assistência à saúde pública. Requer múltiplas ações nas áreas de saúde e educação, principalmente, previstas nas políticas públicas, além da personalização e humanização na atenção ao usuário(17).

A abordagem da adesão deve ser diferente em cada faixa etária, valorizando atentamente necessidades e dificuldades apresentadas pelos hipertensos. Pessoas de baixa escolaridade podem ter menor desempenho no autocuidado por não compreenderem as orientações do profissional de saúde ou por não terem habilidade leitora para entender a prescrição ou ainda por dificuldades na sua interpretação, o que conflui com a associação entre baixa adesão e escolaridade encontrada ${ }^{(15,19)}$.

Entre os participantes do estudo, havia muitos idosos com baixa renda, LFS e adesão. A renda mostrou associação com abandono ou seguimento inadequado do tratamento, decorrente da não rara necessidade de comprar fármacos indisponíveis no Sistema Único de Saúde (SUS), alimentação adequada à dieta prescrita e exames de acompanhamento, o que pode gerar ou retardar o diagnóstico de comorbidades/complicações, potencializadas na presença de baixo letramento(16,20).

Além disso, requisitam-se políticas de acesso a serviços de saúde, acompanhamento 
profissional, medidas tecnológicas para controle pressórico, acesso a fármacos modernos. Para isso, urge eleger estratégias de sensibilização de indivíduos, famílias e comunidade, ampliando interesses e capacidades na compreensão do seu processo saúde-doença-cuidado(16).

Diante disso, é prudente que a equipe de saúde conheça o nível de adesão dos usuários, são disponíveis e cientificamente divulgados instrumentos de fácil execução, capazes de fazer essa mensuração. Dois desses são usados neste estudo (QATHAS, MMAS-8). A mensusação do LFS parece ser um indicador importante, no contexto da adesão terapeutica e na determinação de resultados esperados, para intervenções relacionadas à educação em saúde, orientações sobre o autocuidado e prevenção de complicações e do abandono ao tratamento. Tais istrumentos certamente podem facilitar a elaboração de planos terapêuticos ${ }^{(17,20-21)}$.

O LFS é um importante indicador, por ser estratégico na dimensão do pensamento e cuidado da própria saúde e estar relacionado ao bem-estar individual e satisfação com a vida. Envolve também entender materiais escritos, compreender orientações faladas, associadas com conhecimentos prévios e culturais para a gestão do cuidado e promoção da saúde ${ }^{(18)}$. Há muito em comum entre adesão e LFS, sobretudo, no que tange ao acesso a informações em saúde e desenvolvimento da consciência crítica para a ação sobre a própria vida e o contexto social.

O enfermeiro é o profissional que identifica a necessidade da busca ativa aos pacientes faltosos às consultas, planeja, gerencia, coordena e desenvolve atividades de educação em saúde contribuindo para maior participação e adesão terapêutica ${ }^{(6,9,22)}$. A relação entre esse profissional e o usuário, baseada na educação em saúde, possibilita o aprendizado e melhora o letramento em saúde do paciente, promovendo nele autonomia para a tomada de decisões pertinentes à sua saúde e bem-estar.

Tendo em vista que o baixo LFS pode afetar a interação entre paciente e profissional, nos processos comunicativos, de modo a gerar incompreeção de informações relativas à doença, terapêutica medicamentosa ou não medicamentosa e cuidados, faz do LFS importante condutor da boa comunicação terapêutica, fortalecendo vínculos de empatia e credibilidade, tão importantes no adoecimento crônico ${ }^{(20-23)}$.

\section{CONCLUSÃO}

A maioria dos participantes tinha LFS inadequado. Tal homogeneidade nos achados restou na associação não significativa entre adesão e LFS. Apenas as variáveis faixa etária e escolaridade tiveram associação estatística significativa com o LFS, que, em números absolutos, foi mais inadequado entre os mais velhos e com menos escolaridade. Na aplicação do instrumento QATHAS, a maioria aderia ao tratamento, mas poucos em seu nível máximo e mostrou significativa apenas com pressão arterial. Com o MMAS-8, foi constatada menor adesão, certamente pelo fato da sua abordagem específica ao tratamento farmacológico e demonstrou associação significativa somente com a idade.

Por se tratar de população de pouca escolaridade comparada às populações de países desenvolvidos, o instrumento foi elaborado e aplicado. É importante salientar que pesquisas sobre LFS no Brasil ainda são recentes, não tendo sido elaborado nenhum instrumento adaptado à realidade brasileira, emergindo, assim, a necessidade da criação de instrumentos brasileiros para avaliar o LFS.

Explorar os fatores condicionantes e determinantes do LFS que se relacionam com a adesão, pode ampliar o conhecimento e subsidiar intervenções específicas, a serem incorporadas na prática de enfermagem, sendo relevante conhecer melhor a relação LFS e adesão aos diversos contextos culturais e de cuidado nas cronicidades em saúde.

As contribuições à prática clínica de enfermagem à aplicação dos instrumentos e associação com a adesão e o LFS, embora sem associação estatística significativa, referem-se à sua possibilidade em direcionar o planejamento de ações de educação em saúde para a melhora do letramento e adesão terapêutica antihipertensiva. No ensino, sugere-se a qualificação de profissionais sobre o uso dos instrumentos de mensuração do LFS e adesão terapêutica, como subsídios da prática clínica de enfermagem na atenção primária à saúde.

O estudo, em seu diagnóstico situacional, poderá ainda contribui na sensibilização de gestores sobre a importância de olhar para essa temática do LFS e adesão terapêutica e sua inserção em políticas públicas no Brasil. Espera-se ser oportuno que o diagnóstico situacional aqui exposto possa subsidiar ações de educação em 
saúde e planos de cuidados individualizados no contexto de cada hipertenso. A união entre gestão, profissionais de saúde e usuários dos serviços é de grande valia no fortalecimento das ações de promoção da saúde e prevenção das complicações.

Os resultados sugerem a necessidade de elaboração de materiais educativos, cuja leitura seja adequada ao nível de letramento dos usuários do SUS, de modo que facilite o processo de adesão. Ademais, as medidas de promoção da saúde e prevenção de condições crônicas devem ter como metas (i) o diagnóstico situacional do letramento em saúde; (ii) melhor interação profissional-usuário, enfatizando a comunicação oral e escrita; e (iii) incluir a comunidade e a família no processo de cuidado.

Foi limitação da pesquisa a aplicação do instrumento de letramento enquanto questionário, pois algumas das palavras apresentadas requeriam habilidades maiores de interpretação, o que pode ter interferido na compreensão do significado de algumas palavras pelos hipertensos. Tal situação foi minimizada pelo olhar atento da pesquisadora e sua disponibilidade em explicar significados e facilitar a compreensão. Essa limitação ratifica a necessidade de instrumentos construídos para a realidade brasileira.

\section{REFERÊNCIAS}

1 - Malta DC, Bernal RTI, Andrade SSCA, Silva MMA, Velasquez-Melendez G. Prevalência e fatores associados com hipertensão arterial autorreferida em adultos brasileiros. Rev Saúde Pública 2017;51(supl 1):1s-11s. DOI: $\underline{10.1590 / \mathrm{s} 1518-8787.2017051000006}$

2 - Becho AS, Oliveira JLT, Almeida GBS. Dificuldades de adesão ao tratamento por hipertensos de uma unidade de atenção primária à saúde. Rev APS 2017;20(3):349-59. DOI: $\underline{10.34019 / 1809-8363.2017 . v 20.15608}$

3- Vrijens B, Antoniou S, Burnier M, Sierra A, Volpe M. Current situation of medication adherence in hypertension. Front Pharmacol. 2017; 8(100):1-8. DOI: 10.3389/fphar.2017.00100

4 - Moura AA, Godoy S, Cesarino CB, Mendes IAC. Fatores da não adesão ao tratamento da hipertensão arterial. Enferm Global 2016;15(3):139. DOI: $\underline{10.6018 / \text { eglobal.15.3.219601 }}$
5 - Marques SRL, Escarce AG, Lemos SMA. Health literacy and self-rated health in adults primary care patients. CoDAS 2018;30(2):e20170127. DOI: 10.1590/2317$\underline{1782 / 20182017127}$

6 - Martins NFF, Abreu DPG, Silva BT, Semedo DSRC, Pelzer MT, lenczak FS. Letramento funcional em saúde e adesão à medicação em idosos: Revisão integrativa. Rev Bras Enferm. 2017;70(4):868-74. DOI: 10.1590/0034-71672016-0625

7 - Machado ALG, Guedes IH, Costa KM, Borges FM, Silva AZ, Vieira NFC. Perfil clínicoepidemiológico e adesão ao tratamento de idosos com hipertensão. Rev Enferm UFPE 2017;11(12):4906-12. DOI: 10.5205/1981-8963v11i12a22996p4906-4912-2017

8 - Malachias MVB, Póvoa RMS, Nogueira AR, Souza D, Costa LS, Magalhães ME. 7ạ Diretriz Brasileira de Hipertensão Arterial: Capítulo 3 Avaliação Clínica e Complementar. Arq Bras Cardiol. 2016;107(3 supl 3):14-7. DOI: 10.5935/abc. 20160153

9 - Borges FM, Silva ARV, Lima LHO, Almeida PC, Vieira NFC, Machado ALG. Letramento em saúde de adultos com e sem hipertensão arterial. Rev Bras Enferm. 2019;72(3):646-53. DOI: 10.1590/0034-7167-2018-0366

10 - Herdman TH, Kamitsuru SE. Nanda International. Diagnósticos de enfermagem da NANDA-I: Definições e classificação 2018-2020. 11a ed. Porto Alegre: Artmed; 2018.

11 - Brasil. Ministério da Saúde. Resolução no 466, de 12 de dezembro de 2012. Dispõe sobre diretrizes e normas regulamentadoras de pesquisas envolvendo seres humanos. Brasília: CNS; 2012.

12 - Miot HA. Tamanho da amostra em estudos clínicos e experimentais. J Vasc Bras. 2011;10(4):275-8. DOI: 10.1590/S1677$\underline{54492011000400001}$

13 - Rodrigues MTP, Moreira TMM, Andrade DF. Elaboration and validation of instrument to assess adherence to hypertension treatment. Rev Saúde Públ. 2014;48(2):232-40. DOI: 10.1590/S0034$\underline{8910.2014048005044}$ 
8|Girão AC, Moreira TMM, Silva JR, et al.

14 - Oliveira-Filho AD, Barreto-Filho JA, Neves SJF, Lyra Junior DP. Association between the 8-item Morisky Medication Adherence Scale (MMAS-8) and blood pressure control. Arq Bras Cardiol. 2012;99(1):649-58. DOI: 10.1590/S0066$\underline{782 \times 2012005000053}$

15 - Apolinario D, Braga R, Magaldi R, Busse A, Campora F, Brucki $S$, et al. Short assessment of health literacy for portuguese-speaking adults. Rev Saúde Pública 2012;46(4):702-11. DOI: $\underline{10.1590 / S 0034-89102012005000047}$

16 - Santos MIPO, Portella MR. Condições do letramento funcional em saúde de um grupo de idosos diabéticos. Rev Bras Enferm. 2016;69(1):156-64. DOI: 10.1590/0034$\underline{7167.2016690121}$

17 - Raymundo ACN, Pierin AMG. Adesão ao tratamento de hipertensos em um programa de gestão de doenças crônicas: Estudo longitudinal retrospectivo. Rev ESC Enferm USP 2014;48(5):811-9. DOI: 10.1590/S0080$\underline{6234201400005000006}$

18 - Farzaneh D, Shahzad P, Reza N. The effects of self-management education tailored to health literacy on medication adherence and blood pressure control among elderly people with primary hypertension: A randomized controlled trial. Patient Educ Couns. 2020;103(2):336-42. DOI: $10.1016 /$ j.pec.2019.08.028

19 - Salles ALO, Sampaio CEP, Pereira LS, Malheiros NS, Gonçalves RA. O enfrmeiro e a questão da adesão do paciente ao tratamento da pressão arterial sistêmica. Rev Enferm UERJ 2019;27:e37193. DOI: 0.12957/reuerj.2019.37193

20 - Oscalices MIL, Okuno MFP, Lopes MCBT, Batista RE, Assayag CCRV. Literacia em saúde e adesão ao tratamento de pacientes com insuficiência cardíaca. Rev Esc Enferm USP 2019;53:e03447. DOI: $10.1590 /$ s1980$\underline{220 \times 2017039803447}$

21 - Saqlain M, Riaz A, Malik MN, Khan S, Ahmed A, Kamran S, Ali H. Medication adherence and its association with health literacy and performance in activities of daily livings among elderly hypertensive patients in Islamabad, Pakistan. Medicina 2019;55(5):163. DOI: $\underline{10.3390 / \text { medicina55050163 }}$
22 - Lor M, Koleck TA, Bakken S, Yoon S, Dunn NAM. Association Between Health Literacy and Medication Adherence Among Hispanics with Hypertension. J Racial Ethn Health Disparities 2019;6(3):517-24. DOI: 10.1007/s40615-01800550-z

23 - Mafutha N, Mogotlane S, Swardt $H$. Development of a Hypertension Health Literacy Assessment Tool for use in primary healthcare clinics in South Africa, Gauteng. Afr J Prim Health Care Fam Med. 2017; 9(1):a1305. DOI: 10.4102/phcfm.v9i1.1305

\section{Editores Responsáveis:}

Patrícia Pinto Braga

Daniel Nogueira Cortez

Nota: Extraído da dissertação de mestrado do Programa de Pós-Graduação em Cuidados Clínicos em Enfermagem e Saúde da Universidade Estadual do Ceará, intitulada: "Análise da associação da adesão terapêutica da hipertensão arterial sistêmica e o letramento funcional em saúde na atenção primária".

Recebido em: $17 / 12 / 2020$

Aprovado em: 17/05/2021 